\title{
A FUNCTIONAL DECLINE MODEL FOR PREVALENT COHORT DATA
}

\author{
XINHUA LIU \\ HIV Center for Clinical and Behavioral Studies, New York State Psychiatric Institute and Columbia University. \\ New York, NY 10032, U.S.A. \\ WEI-YANN TSAI \\ Division of Biostatistics, Columbia University School of Public Health, New York, NY 10032, U.S.A.
}

AND

YAAKOV STERN

Gertrude H. Sergievsky Center, Departments of Neurology and Psychiatry, Columbia University College of Physicians and Surgeons, New York, NY 10032, U.S.A

\section{SUMMARY}

Longitudinal designs are often used for studying the natural history of diseases. Data sets typically consist of short series of repeated measures on prevalent cases. We propose a growth model approach to the analysis of follow-up data to describe functional decline and associated risk factors in disease progression. We illustrate the model with an application to longitudinal data that describe the time-evolution of cognitive decline in a cohort of patients with Alzheimer's disease.

\section{INTRODUCTION}

Better understanding of the course of disease progression is important. It is essential for clinicians, as well as patients and their families, to have accurate prognostic information. Natural history studies are conducted to evaluate progressive disorders and investigate patterns of functional decline, time to certain endpoints such as severe functional deficit, nursing home or death, and potential predictors of rapid progression.

It is known that linear models are not sufficient to portray functional decline in disease progression. Non-linear models may be more appropriate, and deserve more consideration. For example, cognitive deficits in Alzheimer's disease typically begin with memory disturbances and progress to a point where no abilities are spared. To describe the pattern of decline, Brooks et al. ${ }^{1}$ proposed a trilinear model with an initial period of stability before detectable decline on a cognitive test score, a period of decline, and a final period of stability with no further detectable decline. Morris and his colleagues ${ }^{2}$ also showed that the rate of change of test scores varied with level of cognitive impairment of the patients. These observations suggest the study of a family of non-linear models. Non-linear growth models, often characterized by S-shape curves, are well known and have had wide use in many fields such as biology, chemistry, economics and medical 
research. When patients' test scores, measured over time, show a pattern of S-shape decline, the fit of a proper growth model to the longitudinal data may help to describe and predict disease progression. With such a model, we can identify a typical rapid progression and can easily estimate the time to reach a specific value of the test score.

In a longitudinal study design, it is common among subjects recruited in a prevalent cohort that they are at different stages of their disease. They are then examined regularly over the followup period. The longitudinal data can contain many short series of functional measures, since a number of subjects may be examined only for few visits. Non-linear curve fitting of this kind of data can be complicated, especially when the time of disease onset is unclear.

The purpose of this paper is to extend non-linear growth models ${ }^{3}$ so that we can use them to characterize various shapes of functional decline in disease progression as well as to explore effects of possible risk factors on the functional decline pattern. The method described in Section 2 applies to data from a prevalent cohort where subjects' disease onset times are unclear. Section 3 presents an application of the method followed by a discussion in Section 4 .

\section{METHOD}

\subsection{Basic model}

Suppose that $Y(T)$ is a stochastic process that takes values in $R^{+}$, and measures occur at time $T=t_{0}, \ldots, t_{n}$ with equal time intervals such that $t_{k+1}-t_{k}=c$ for all $k$. Here $c$ is a known constant. Let $Y_{k}=Y\left(t_{k}\right)$ and $A_{k}=Y_{k+1}-Y_{k}$ denote the difference between two consecutive measures, $k=0,1, \ldots, n$. We specify our model by the first two conditional moments:

$$
\begin{aligned}
E\left(A_{k} \mid Y_{0}, \ldots, Y_{k}\right) & =G\left(Y_{k} ; \theta\right) \\
\operatorname{var}\left(A_{k} \mid Y_{0}, \ldots, Y_{k}\right) & =\tau
\end{aligned}
$$

where $G(\cdot)$ is a differentiable function and $\theta$ and $\tau$ are unknown parameters. $\tau$ is a positive constant, while $\theta$ can be a vector that determines the shape of the time series $\left\{E\left(Y_{k+1} \mid Y_{k}\right)\right\}$ generated by the model.

Growth models are popular in describing S-shaped growth. They can be specified by a differential equation that assumes that the growth rate, derivative of size, is proportional to the present size and the future amount of growth. A solution of a specific differential equation is a function of time and usually is a non-linear curve. Examples include logistic models, Gompertz models and monomolecular models, that we can unify by Von Bertalanffy's model ${ }^{3}$,

$$
\begin{aligned}
y(t) & =\left(\alpha^{1-m}-(1-m) \exp (-\beta(t-u))\right)^{1 /(t-m)} & & \text { if } m \neq 1 \\
& =\alpha \exp (-\exp (-\beta(t-u))) & & \text { if } m=1
\end{aligned}
$$

or

$$
\begin{array}{rlrl}
\frac{d y(t)}{d t} & =\beta y \frac{\left((\alpha / y)^{1-m}-1\right)}{1-m} & & \text { if } \mathrm{m} \neq 1 \\
& =\beta y \log (\alpha / y) & \text { if } m=1 .
\end{array}
$$

When $\beta>0, y(t)$ increases with $t$; and when $\beta<0, y(t)$ decreases with $t$. Figure 1 shows some of the curves with $\alpha=2$ and $\beta=1$ for various $m$ and $u$. $m=0$ produces a monomolecular curve, 


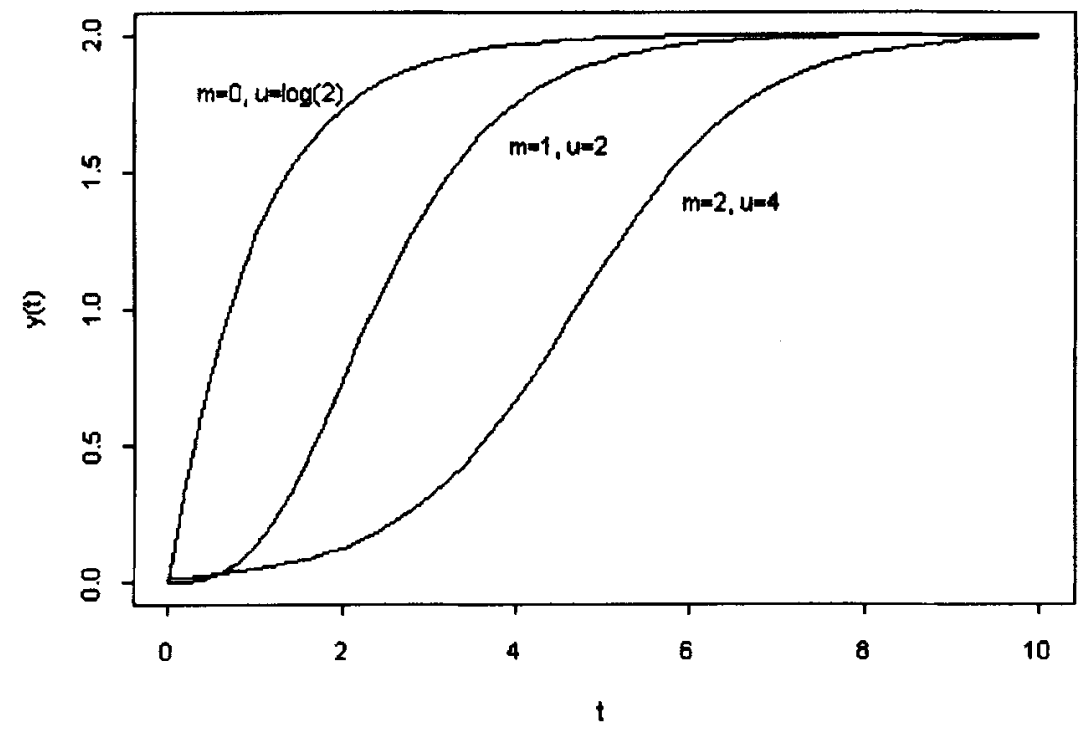

Figure 1. Growth curves from Von Bertalanffy's model

$m=1$ a Gompertz curve, and $m=2$ a logistic curve. By assuming that the observed data $y_{i}$ on time $t_{i}, i=1, \ldots, n$, are uncorrelated and that

$$
y_{i}=y\left(t_{i}\right)+\varepsilon_{i}
$$

with $E\left(\varepsilon_{i}\right)=0$ and $\operatorname{var}\left(\varepsilon_{i}\right)=\sigma^{2}$, we can use conventional non-linear curve fitting procedures to fit the growth curve specified by the time function $y(t)$ to the data $\left(y_{i}, t_{i}\right)$ with $t_{i}$ known.

In a prevalent cohort, repeated measures occur regularly over the follow-up period, but the follow-up time may not relate consistently to the time since disease onset, and the time since disease onset is often hard to estimate precisely. In this case, we cannot directly apply conventional non-linear curve fitting procedures, and it can be very complicated to fit a non-linear curve of the explicit time function.

In developing an alternative method for analysing this type of data, we note that with subjects examined at a constant time interval, we can interpret the difference between functional measures at two consecutive visits as the rate of change over the period, and that may depend on the value of a previous functional measure. Secondly, when we replace the differential equation of a growth curve with a difference equation, we can also generate non-linear curves. Furthermore, the differential or difference equation does not involve a time variable directly. To use a difference equation, we require equal length time intervals. This is typically feasible in a follow-up study.

To specify the function $G(\cdot)$ in (1), we adopt a form similar to Von Bertalanffy's model ${ }^{3}$

$$
\begin{array}{rlrl}
G\left(Y_{k} ; \boldsymbol{\theta}, m\right) & =\beta \frac{\left(Y_{k}-\alpha_{0}\right)}{1-m}\left(\left(\frac{\alpha_{1}}{Y_{k}-\alpha_{0}}\right)^{1-m}-1\right) & \text { if } m \neq 1 \\
& =\beta\left(Y_{k}-\alpha_{0}\right)\left(\log \left(\alpha_{1}\right)-\log \left(Y_{k}-\alpha_{0}\right)\right) \quad \text { if } m=1
\end{array}
$$

where $\theta=\left(\alpha_{0}, \alpha_{1}, \beta\right)$. Note that $G\left(Y_{k}\right)=0$ at $Y_{k}=\alpha_{0}$ or $Y_{k}=\alpha_{0}+\alpha_{1}$, indicating no change at that point. When $m>0, Y_{k}$ may take on a value between $\alpha_{0}$ and $\alpha_{0}+\alpha_{1}$. In this case, we can view 
$\alpha_{0}$ and $\alpha_{0}+\alpha_{1}$ as possible 'bounds' that $Y_{k}$ approaches, the average conditional change $E\left(A_{k} \mid Y_{k}\right)$ vanishes. The parameter $\beta$ characterizes dynamic change, and its sign determines the direction of the average conditional change. The power parameter $m$ indicates various models. For $m=0$, $E\left(A_{k} \mid Y_{k}\right)=\beta\left(\alpha_{0}+\alpha_{1}-Y_{k}\right)$, corresponding to a monomolecular model with only one bound. When $Y(T)$ is a Gaussian process, it is an autoregression model (AR-1), since we can rewrite (1) as $E\left(Y_{k+1} \mid Y_{k}\right)=\alpha+\beta^{*}\left(Y_{k}-\alpha\right)$ with $\alpha=\beta\left(\alpha_{0}+\alpha_{1}\right)$ and $\beta^{*}=1-\beta$. When $m=2, E\left(A_{k} \mid Y_{k}\right)=$ $\left(\beta / \alpha_{1}\right)\left(Y_{k}-\alpha_{0}\right)\left(\alpha_{0}+\alpha_{1}-Y_{k}\right)$, corresponding to a logistic model. While $m \rightarrow 1$, we have $E\left(A_{k} \mid Y_{k}\right)=\beta\left(Y_{k}-\alpha_{0}\right) \log \left(\alpha_{1} /\left(Y_{k}-\alpha_{0}\right)\right)$, corresponding to a Gompertz model. The parameter $m$ can take other values as well.

When $m>0$, there exists a point $Y_{c}$ where the rate of change is at its maximum, such that $\mathrm{d} G(Y) / \mathrm{d} Y=0$ at $Y=Y_{c}$. Solving the equation, we have

$$
\begin{aligned}
Y_{c} & \left.=\alpha_{0}+\alpha_{1} m^{1 /(1-m)}\right) & & \text { if } m \neq 1 \\
& =\alpha_{0}+\alpha_{1} \mathrm{e}^{-1} & & \text { if } m=1 .
\end{aligned}
$$

\subsection{Estimation}

Let $\mathbf{y}_{\mathbf{i}}=\left(y_{i, 0}, \ldots, y_{i, n_{i}}\right)$ denote a series of functional measures for the $i$ th subject in the follow-up study. It is the $i$ th realization of the process $Y(T)$ with $T_{i} \in\left\{t_{i, k}\right\}, t_{i, k+1}-t_{i, k}=c, k=$ $0,1, \ldots, n_{i}-1$, where $c$ is a known constant, but we cannot always observe $t_{i, 0}$. Then we can obtain $\mathbf{A}_{\mathbf{i}}=\left(A_{i, 0}, \ldots, A_{i, n_{i}-1}\right)$ such that $A_{i, k}=y_{i, k+1}-y_{i, k}, k=0, \ldots, n_{i}-1 ; i=1, \ldots, N$. Suppose that $y_{i} s$ are independent and $\bigcup_{i=1}^{N}\left(t_{i, 0}, t_{i, n_{i}}\right)$ covers the range of $T$. The residuals, $r_{i, k}=A_{i, k}-E\left(A_{i, k} \mid y_{i, k}\right)$, are uncorrelated and thus we have

$$
E\left(r_{i, k} r_{j, h}\right)= \begin{cases}0 & i \neq j \text { or } k \neq h \\ \tau & i=j \text { and } k=h .\end{cases}
$$

Following Godambe, ${ }^{4}$ Zeger and Qaqish, ${ }^{5}$ we adopt a quasi-likelihood approach to estimate the model parameters. The estimating functions for $\theta$ and $m$ take the form

$$
\begin{aligned}
& S_{1}(\theta, m)=\sum_{i=1}^{N} \sum_{k=0}^{n_{i}-1} \frac{\partial E\left(A_{i, k} \mid Y_{i, k}\right)}{\partial \theta} \operatorname{var}^{-1}\left(A_{i, k} \mid Y_{i, k}\right) r_{i, k} \\
& S_{2}(\theta, m)=\sum_{i=1}^{N} \sum_{k=0}^{n_{i}-1} \frac{\partial E\left(A_{i, k} \mid Y_{i, k}\right)}{\partial m} \operatorname{var}^{-1}\left(A_{i, k} \mid Y_{i, k}\right) r_{i, k} .
\end{aligned}
$$

We can obtain consistent estimates of $\theta$ and $m$ by solving equations $S_{1}=0$ and $S_{2}=0$ simultaneously. Note that since in (2) $\operatorname{var}\left(A_{i, k} \mid Y_{i, k}\right)$ is a constant, we can exclude it from (6) and (7) when solving the equations.

When $\alpha_{0}$ is known, taking $z_{i, k}=y_{i, k}-\alpha_{0}, A_{i, k}$ is unchanged because $z_{i, k+1}-z_{i, k}=$ $y_{i, k+1}-y_{i, k} . E\left(A_{i, k} \mid z_{i, k}\right)$ is a linear function of $z_{i, k}$ and $z_{i, k}^{m}$, or a linear function of $z_{i, k}$ and $z_{i, k} \log \left(z_{i, k}\right)$, since for any specific $m$,

$$
\begin{aligned}
G\left(z_{i, k} ; \theta, m\right) & =\frac{-\beta}{1-m} z_{i, k}+\frac{\beta \alpha_{1}^{1-m}}{1-m} z_{i, k}^{m}=\alpha_{1}^{*} z_{i, k}+\beta^{*} z_{i, k}^{m} & & \text { if } m \neq 1 \\
& =\beta \log \left(\alpha_{1}\right) z_{i, k}-\beta z_{i, k} \log \left(z_{i, k}\right)=\alpha_{1}^{*} z_{i, k}+\beta^{*} z_{i, k} \log \left(z_{i, k}\right) & & \text { if } m=1 .
\end{aligned}
$$

Here $\theta^{*}=\left(\alpha_{1}^{*}, \beta^{*}\right)$ has a one to one correspondence to $\theta=\left(\alpha_{1}, \beta\right)$. Solving the equation $S_{1}=0$ may actually produce a least squares estimate of $\theta^{*}(m)$. Therefore we can apply the routines in 
popular statistical packages such as SAS, SPSS and GLIM to estimate $\theta^{*}(m)$ for a specific $m$ and we can embed them in a program for seeking $\hat{m}$ by numerically solving $S_{2}=0$. Finally, we can obtain an estimate of $\theta$ by taking a transformation from the LS estimate of $\theta^{*}$.

We can estimate the scale parameter $\tau$ by the moment method as mean square error; that is,

$$
\hat{\tau}=\frac{\sum_{i=1}^{N} \sum_{k=0}^{n_{i}-1} r_{i, k}^{2}}{\sum_{i=1}^{N} n_{i}} .
$$

Based on the proposition of Zeger and Qaqish, ${ }^{5}$ we can calculate approximate 95 per cent confidence intervals for $\boldsymbol{\eta}=(\boldsymbol{\theta}, m)$. Under some weak conditions, $\sqrt{ } N_{\eta}(\hat{\eta}-\eta)$ converges to a Gaussian random vector with mean 0 and variance $\Sigma$, here $N_{\eta}=\sum_{i=1}^{N} n_{i}$. We can estimate the asymptotic variance of $\hat{\eta}$ by replacing $\boldsymbol{\eta}$ with its estimate in the following formula

$$
\hat{\Sigma}=\left(\begin{array}{ll}
E S_{1}^{t} S_{1} & E S_{1}^{t} S_{2} \\
E S_{2} S_{1} & E S_{2}^{2}
\end{array}\right)^{-1} .
$$

When we specify parameters $m$ and $\boldsymbol{\theta}$ and we have an initial value $y_{0}$, we may generate a time series by calculating $\hat{Y}_{k+1}=E\left(Y_{k+1} \mid \hat{Y}_{k}\right)=\hat{Y}_{k}+G\left(\hat{Y}_{k} ; \theta, m\right)$ for $k=0,1, \ldots$, to describe the average functional change over time. We can then estimate typical time $t_{n}$ to a certain value of $Y$ from the curve. Therefore we can make predictions about future change. We can estimate the point $Y_{c}$ associated with maximum rate of change, if it exists, either from the curve or by formula (4). Since $Y_{c}$ is a function of parameters $\alpha_{0}, \alpha_{1}$ and $m$, when the variance of the parameter estimates are available, we can calculate a standard error of estimate $\hat{Y}_{c}$ by the well known delta method; approximate 95 per cent confidence intervals are simply $\hat{Y}_{c} \mp 1.96$ s.e. $\left(\hat{Y}_{c}\right)$. In the case that $\alpha_{0}$ and $\alpha_{1}$ are known, for $0<m \neq 1$,

$$
\text { s.e. }\left(\hat{Y}_{c}\right) \approx \alpha_{1}\left(\frac{1-m+m \log (m)}{(1-m)^{2}}\right) m^{m /(1-m)} \text { s.e. }(\hat{m}) .
$$

We can obtain an estimate of s.e. $\left(\hat{P}_{c}\right)$ by replacing $m$ with its estimate.

\subsection{Extended model}

When previous studies suggest a potential predictor of rapid progression of a disease, it is important to understand further its role in the disease course and describe its effect on functional decline. Let $X$ denote a risk factor that may have an impact on a patient's functional process $Y(T)$. We can extend the basic growth model to include $X$ so that we can assess its effect on the shape of the curve.

We can establish the extension in a flexible way, since the first moment $E\left(A_{k} \mid Y_{k}, X\right)=G\left(Y_{k}, X\right)$ can take various forms. For example, to test the effect of $X$ on the rate of change, we may consider the model

$$
G\left(Y_{k}, X ; \gamma, \theta, m\right)=R(X ; \gamma) G\left(Y_{k} ; \theta, m\right)
$$

where $G\left(Y_{k} ; \theta, m\right)$ is the basic model defined by (3), and the function $R(X ; \gamma)$ reflects the relative rate of change due to $X$. Taking a commonly used simple form, $R(X ; \gamma)=\exp (\gamma X)$, we can interpret $\gamma$ as a relative rate of functional change caused by the increase of a unit of $X$. When $X$ is a binary variable that takes values of 0 or $1, \exp (\gamma)$ is the ratio of the conditional average change in the two groups indicated by $X$. In principle, $X$ can be in vector form. The extension of the model is straightforward. 
Table I. Estimated model parameters (s.e.) and 95 per cent confidence intervals for a basic model of mMMS progression, and an extended model that incorporates age at onset as a covariate.

\begin{tabular}{lrrrr}
\hline Parameter & \multicolumn{2}{c}{ Basic model } & \multicolumn{2}{c}{ Extended model } \\
\hline$\beta$ & -0.1724 & $(0.0210)$ & -0.1629 & $(0.0221)$ \\
& $(-0.2118$, & $-0.1330)$ & $(-0.2062$, & $-0.1247)$ \\
$m$ & 0.8631 & $(0.2943)$ & 1.1317 & $(0.3434)$ \\
& $(0.2863$, & $1.4399)$ & $(0.4586$, & $1.8048)$ \\
$\gamma$ & & & 0.3466 & $(0.1132)$ \\
& & $(0.1247$, & $0.5685)$ \\
$\tau$ & 23.8197 & \multicolumn{2}{c}{23.4572} \\
\hline
\end{tabular}

We can also treat $X$ as a time dependent variable. Let $X(T)$ denote an independent process that interferes with $Y(T)$, then $E\left(A_{k} \mid Y_{k}, X_{k}\right)=G\left(Y_{k}, X_{k} ; \gamma, \theta, m\right)$. In this case, we can still use the above models by replacing $X$ with $X_{k}$.

\section{APPLICATION}

We obtained the data from a multi-centre study of disease course in Alzheimer's disease. The Predictors Study ${ }^{6}$ has followed a cohort of 236 patients with probable Alzheimer's disease from New York, Baltimore and Boston for up to four years. The broad objective of the study is to develop a predictor model of the natural history of Alzheimer's disease. Patients were recruited in the early stage of the disease, and underwent neurological, neuropsychological, psychiatric and functional assessments at baseline and at repeated six-month intervals.

A modified Mini-Mental State Examination (mMMS) was used to measure patients' global intellectual function. The maximum score is 57 , indicating normal cognitive ability, and minimum score is 0 , indicating total loss of cognitive ability. Previous studies have suggested that a patient's score initially remains relatively stable at the higher level, then declines continuously until reaching another period of stability, with no further decline detectable. ${ }^{1,2}$ We have observed a similar phenomenon in our data set in which there were 1049 mMMS test scores available on 218 subjects who had completed at least two consecutive visits and had been followed for 6 to 54 months. Observed scores ranged from 0 to 57 . The change between two consecutive visits ranged from -29 to 8 . Patients with higher mMMS test scores at baseline tended to have smaller declines, while patients with lower test scores seemed to decline faster. Those patients with zero scores were experiencing the last stage of the disease, since a period of stable minimum score was often followed by death. These observations encouraged us to apply a basic growth model to the mMMS test scores. The average conditional change in the six month interval is described by (3) with $\alpha_{0}=0$ and $\alpha_{1}=57$. Estimated parameters of the basic model appear in Table I.

The estimate of $\beta$ is negative, indicating a decline in the test score. The estimate of the power parameter $m$ is close to 1, suggesting that the pattern of decline is similar to a Gompertz model. The parameter $\tau$, which characterizes the conditional variance of the changes, is also the variance of residuals as defined in Section 2.2. Taking its square root, we have the standard deviation of residuals; 4.88 is the estimate for the basic model. 


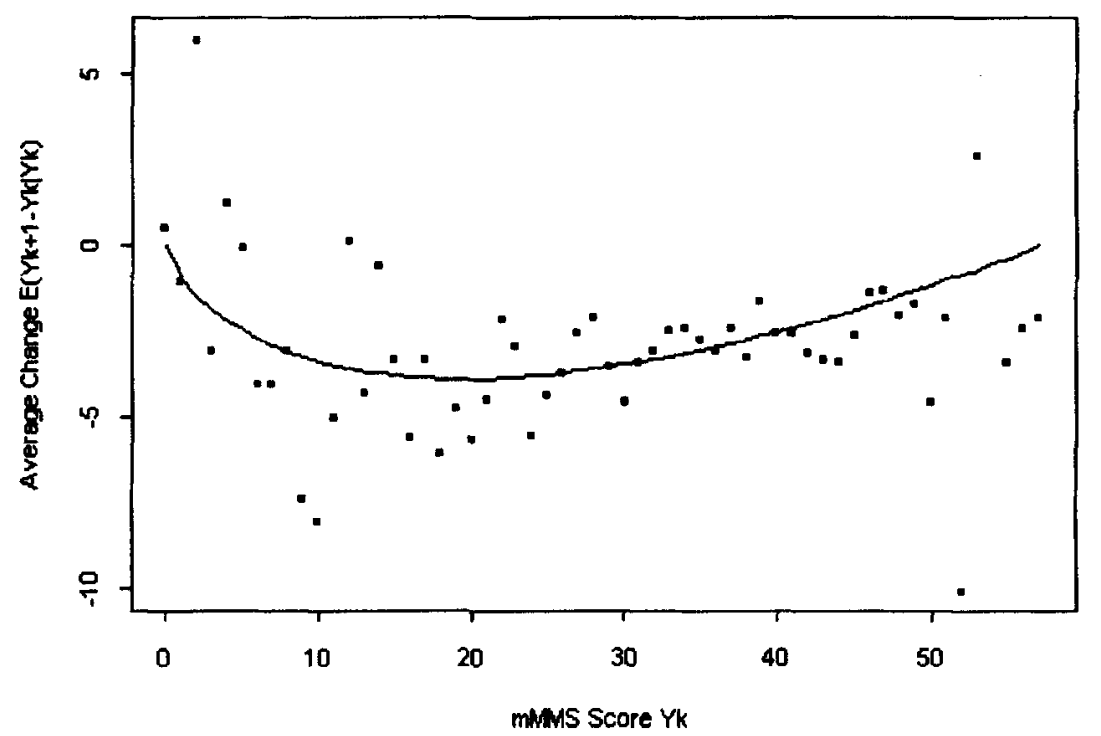

Figure 2. Conditional average change of mMMS score over six-month interval in 218 Alzheimer's disease patients

For each given score, we calculated the associated conditional average changes in a six-month interval by two methods: one is based on the model, the other is simply the mean of the observed changes. Figure 2 demonstrates that the smooth curve generated from the model seems to fit the empirical estimates fairly. The latter fluctuate markedly on the low and high given scores for which there are very few observations available. We can see from the curve that as the score decreases, the amount of decline gradually increases until the score drops to about $19\left(Y_{c}=19 \cdot 4\right.$, 95 per cent $\mathrm{CI}: 12 \cdot 6-26.3$ ), then it converges to zero as the score reaches its minimum. The pattern is compatible with that noted in another cohort study where the annual decline rates of MMS scores were displayed as a function of the average scores during the observation period. ${ }^{2}$

Taking an initial value $y_{0}=56$, we use the model to generate a curve of mMMS scores (Figure 3). From the curve we may roughly estimate the period of decline as about 15 years.

There is no pattern on the residual versus score plot (Figure 4), and 95 per cent of residuals for the basic model are within two standard deviations from mean zero, indicating that the basic model fits the data well. Although the model explains the majority of the data, the fact that there are some large residuals indicates some unexplained rapid decline and suggests that further application of the extended model to introduce other predictor variables may be useful.

Previous studies have suggested that early onset of Alzheimer's disease (before age 65) is characterized by faster clinical progression and is associated with decreased relative survival. Recently Jacobs et al. ${ }^{7}$ provided some longitudinal evidence of more rapid cognitive decline in patients with early onset of Alzheimer's disease. They compared the rate of decline in cognitive measures including mMMS scores for patients in early and late onset groups who had comparable scores at baseline and were followed for two years. We used our model to study further the difference in the rate of cognitive decline between early and late onset groups across the entire disease span.

There were 678 mMMS test scores available for 141 subjects in the late onset group and 361 scores for 74 subjects in the early onset group. We first fit the basic model to the two groups' data separately. We rejected the null hypothesis that the two groups follow the same process 


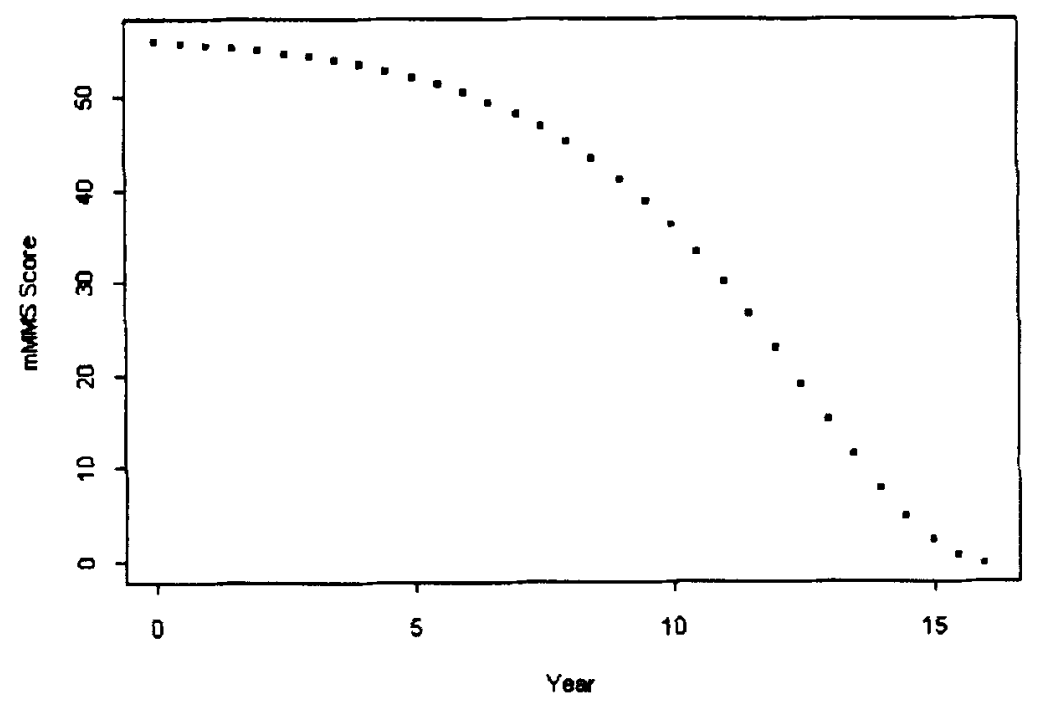

Figure 3. A time series of $m M M S$ score $\left\{E\left(Y_{k+1} \mid Y_{k}\right\}\right.$ generated by model with initial score $Y_{0}=56$
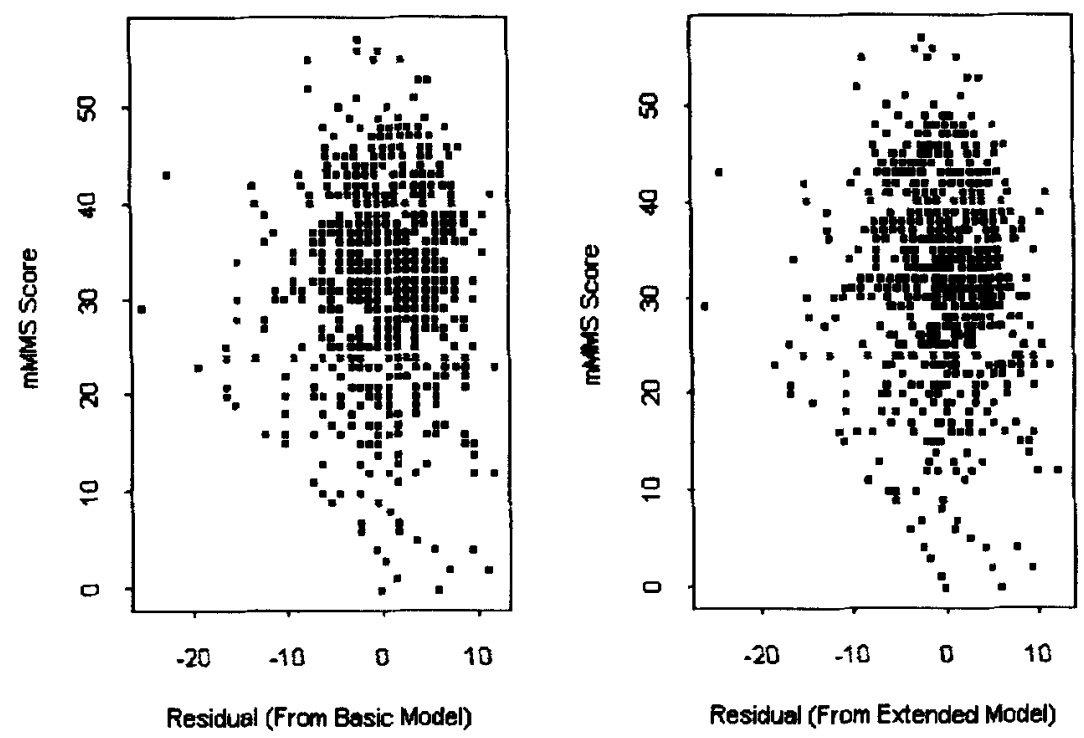

Figure 4. Residual versus score plot

$\left(\chi^{2}=6.77\right.$, d.f. $\left.=2, p=0.03\right)$. We did not, however, reject the null hypothesis of equal power parameter ( $m$ in formula (3)) in the two groups $\left(\chi^{2}=1 \cdot 88, \mathrm{~d} f .=1, p=0 \cdot 17\right)$. This suggested consideration of an extended model such as (10), where we treat $X$ as an indicator variable, 1 for the early onset group and 0 for the late onset group. Estimated parameters for this extended model appear in Table I. It is clear that the decline pattern is similar in both groups since the estimate of $\beta$ is negative and they share the same power parameter. The estimate of $\gamma$ is positive 
and significantly different from zero $(p<0.05)$. This indicates that for each given score, the average conditional change of mMMS scores over a six-month interval in the early onset group is larger than that in the late onset group. The rate of decline in the early onset group is $\exp (\hat{\gamma})=1.414(95$ per cent CI: 1.133-1.765) times that in the late onset group. This implies more rapid progression and shorter disease course in the early onset group.

The estimate of scale parameter $\tau$ in the extended model is smaller than that in the basic model. Estimated standard deviation of residuals from the extended model is 4.84 . In the residual versus score plot (Figure 4), although the range of spread is similar, the residuals from the extended model are more condensed than those from the basic model, indicating that the extended model fits the data better. There are still a few 'outliers' that the extended model (10) with the single factor of early onset does not explain. The large residuals suggest the utility of exploration of the effects of additional factors that also may relate to decline.

Note that in Table $I$ the power parameter $m$ in both models has an estimated value close to 1 , suggesting a simplification of the models by taking $m=1$.

\section{DISCUSSION}

This paper proposes a method for modelling functional decline patterns, and investigating the effect of possible predictors on these patterns. The presented example of progression of Alzheimer's disease demonstrates the potential utility of this technique.

The proposed method requires several assumptions. First, the basic model assumes that all patients follow the same underlying functional process in disease progression. This means that each subject's functional measures during the follow-up period are a piece of the realization of this process. Patients, however, are not homogenous and they may have different characteristics that may influence disease progression. The better a model reflects an individual subject's specific pattern of functional decline, the more realistic it is. It would also be more helpful in counselling about disease prognosis. The application of the extended model to patients with specific predictor profiles (for example, early versus late onset) is an initial step, since it can tailor the model better to an individual patient. Still, a model that takes into account subject-specific variations in measures over time is useful although more challenging to develop. To meet this goal, we need further studies of subject-specific patterns of functional decline.

The second required assumption for the modelling technique is that the observed functional measures from all the patients in the follow-up study collectively span the entire disease course. To satisfy this assumption, one should recruit into the study patients both in the beginning or very early stage and in the moderate to late stages of the disease; furthermore, the follow-up period should have sufficient length.

The current method applies only to data collected on consecutive visits with a constant time interval. Since missing data (due to absence on scheduled visits or uncompleted tests) may reduce the power of the method, it would be useful to have further development of a non-linear time series model that takes into account missing data. Alternatively, there should be development of a method for modelling functional decline for data with length-varied time intervals.

\section{ACKNOWLEDGEMENTS}

This research was supported by grant T32-MH19139-05 from the National Institute for Mental Health, grant NIAID-5-R29-AI29004-02 from the National Institutes of Health, Federal grant AG07370 and the Charles S. Robertson Gift for Alzheimer's Disease from the Banbury Fund. 


\section{REFERENCES}

1. Brooks, J., Kraemer, H. C., Tanke, E. D. and Yesavage, J. A. 'The methodology of studying decline in Alzheimer's disease', Journal of American Geriatrics Society, 41, 623-628 (1993).

2. Morris, J. C., Edland, S., Clark, C., Galasko, D., Koss, E., Mohs, R., Belle, G. van, Fillenbaum, G. and Heyman, A. 'The consortium to establish a registry for Alzheimer's disease (CERAD). Part IV. Rate of cognitive change in the longitudinal assessment of probable Alzheimer's disease', Neurology, 43, 2457-2465 (1993).

3. Draper, N. and Smith, H. Applied Regression Analysis, 2nd edn. Wiley, New York, 1981.

4. Godambe, V. P. 'The foundations of finite sample estimation in stochastic processes', Biometrics, 72 , 419-428 (1985).

5. Zeger, S. L. and Qaqish, B. 'Markov regression models for time series: A quasi-likelihood approach', Biometrics, 44, 1019-1031 (1988).

6. Stern, Y., Folstein, M., Richards, M., Miller, L., Bylsma, F., Lafleche, G., Marder, K., Bell, K., Sano, M., Devanand, D., Loreck, D., Wootten, J. and Bello, J. 'Multicenter study of predictors of disease course in Alzheimer's disease (the "Predictors study"). I. Study design, cohort description, and intersite comparisons', Alzheimer Disease and Associated Disorders, 7, 3-21 (1993).

7. Jacobs, D., Sano, M., Marder, K., Bell, K., Bylsma, F., Lafleche, G., Albert, M., Brabdt, J. and Stern, Y. 'Age of onset of Alzheimer's disease: Relation to pattern of cognitive dysfunction and rate of decline', Neurology, 44, 1215-1220 (1994). 